\title{
AC 2007-56: TEAM PLAY! INTEGRATING SPORTS INTO THE ENGINEERING CURRICULUM
}

\section{Jennifer Kadlowec, Rowan University}

Jennifer Kadlowec is an Associate Professor of Mechanical Engineering at Rowan University. She received her BS in physics at Baldwin-Wallace College and her MS and PhD in Mechanical Engineering at the University of Michigan. She has been actively involved in ASEE, serving in officer roles in the Mechanics and ERM Divisions.

\section{Howard Pearlman, Drexel University}

Howard Pearlman is an Associate Professor of Mechanical Engineering at Drexel University. He received his BS, MS and $\mathrm{PhD}$ in Mechanical Engineering at Northwestern University. His research interests are in combustion and low-temperature auto-ignition.

\section{Greg Biren, Rowan University}

John Chen, Rowan University John Chen is an Associate Professor of mechanical engineering at Rowan University, where he has been on the faculty since 1998. Prior to that, he was an Assistant Professor at North Carolina A\&T State University, which was part of the SUCCEED engineering education coalition sponsored by the NSF.

\section{Stephanie Farrell, Rowan University}

\section{Anthony Marchese, Rowan University}

\section{Ali Navvab, Gloucester County College}

Ali Navvab is a full time faculty of Engineering at Gloucester County College, Sewell, NJ. He has a Master's degree in Mechanical Engineering from University of Central Florida and Bachelor's degree in Mechanical Engineering from Florida International University.

\section{Robert Sterner, Rowan University}




\title{
Experiments to Teach Engineering Using Sports Applications
}

\begin{abstract}
The context of sporting activities can be an exciting way to teach engineering principles. We are in the process of developing a series of hands-on modules in order to introduce engineering students to mechanical, aerospace, and chemical engineering principles through application to sports and sports performance. The modules allow for students to explore topics such as aerodynamics, mechanics and transport in the context of sports. The modules will be used in a freshman level course focused on engineering measurements, a new senior undergraduate elective course and integrated into other core courses in mechanical and chemical engineering. The purpose of this paper is to describe the experiments that will be conducted by the students, their relation to science and engineering principles found in sports, various measurements and calculations that the students will perform and how the modules fit within the curriculum.
\end{abstract}

\section{Introduction}

Faculty at Rowan University, Drexel University and Gloucester County College are working on an integrated effort to develop effective modules for teaching engineering from an applied, multidisciplinary point of view. The basis of the project is the fact that the world of sports provides for an exciting basis to study multidisciplinary engineering principles and that most students can relate to sporting activities in some way or another, either as a participant or spectator. Over 90 million people in the U.S. over the age of 6 are frequent exercisers or participants in recreational sports. A clear majority of the population (68\% or 170 million people) participated at least once in any of the sports/activities monitored by ASD.[1] Due to the popularity of sports, studying technology and its effect on sports is a good way to teach basic theories but also a way to allow students to bring their designs to the marketplace. "Studying some of the dynamic effects contained in sports, we can introduce all of the dynamic systems that we are trying to teach our students. Students tend to tune out when studying the same old greasy gearbox.'"[2] These ideas were combined with the key features of the Rowan Engineering program, (1) multidisciplinary education through collaborative laboratory and course work; (2) teamwork as the necessary framework for solving complex problems; (3) incorporation of stateof-the-art technologies throughout the curricula; and (4) creation of continuous opportunities for technical communication [3], to develop this project.

The goals of the project are to:

- engage students and improve learning through novel hands-on experimentation,

- generate excitement among undergraduate students by integrating sports and engineering,

- provide undergraduate students with the opportunity to work on projects related to sports and sports technology,

- collaborate with health and exercise science faculty and students, particularly in the study of sports science and related injuries

- host activities for targeted audiences such as K-12 outreach, teacher training and new faculty preparation 
- disseminate lab handouts and course materials that other colleges and universities may be able to implement to introduce biomedical principles in sports

The first component of this project is the development of a series of hands-on experimental modules that introduce scientific principles commonly taught in aerospace, biomedical, chemical and mechanical engineering through their application to sports equipment and sports performance. The modules will be used in a new senior elective course that will also be developed as part of this project and taught at Rowan and Drexel Universities, an introductory engineering course required of all engineering freshmen at Rowan, and a second year engineering courses at Gloucester County College. Effort will also be devoted to the integration of the modules throughout the engineering curriculum at Rowan and Drexel Universities, outreach activities and promote a pipeline for students at the two-year county college to transfer to four-year universities. This paper provides an overview of all of the modules that are under development and how they tie in to courses that will be offered at the three participating institutions. To date, modules 1, 2, 7 and 8 have been developed and tested by undergraduate and high school students.

\section{Module Development}

Module 1 - Mechanics of sporting materials

A wide variety of elastomers and foams are used in sporting equipment from helmet and knee padding to golf and tennis balls, shoe soles and athletic field surfaces. Materials are chosen based on properties such as elastic modulus, hardness and strength in meeting the demands that a given sports component will be subjected to in play.

The objectives are: (1) Perform background research on foam and elastomer materials. (2) Develop experiments to compress a sample of foam or elastomer and measure force and deformation. (3) Use the concepts of stress and strain and use them with Hooke's Law to compute a modulus of elasticity. (4) Compare a variety of materials and determine how the mechanical properties affect the use in design and function.

Measurements and Calculations: Students will place a material sample in a universal testing machine and use an LVDT and load cell to record the displacement and force during the compressive deformation. This data is used to calculate stress and strain and modulus of elasticity.

Engineering Principles: Students apply concepts of stress, strain, deformations, Hooke's Law, and modulus of elasticity, then compare the moduli of a variety of materials and associate these with their function in sports equipment.

Module 2: The sweet spot of a tennis racket, is there more than one?

Ask a tennis player about choosing a racquet and the topic of the sweet spot may arise in the conversation. In a general sense, the sweet spot is the area of the string bed that produces the best combination of feel and power.[4] The most powerful spot on the string bed is that with the greatest coefficient of restitution. The spot with least vibration is found at the node of the first harmonic. Finally, the spot with least shock is found at the center of percussion, hitting here results in a zero net force at the pivot point. All three of these locations can be determined relatively easily through experimentation and application of dynamics and vibrations concepts. 
The objectives of this module are: (1) Perform background research on the sweet spots of a tennis racquet and investigate the effects on play. (2) Develop experiments to measure ball return height of a ball dropped onto a secure racquet and frequency response of a tennis racquet. (3) Calculate the center of percussion of a tennis racquet.

Measurements and Calculations: By securing the racquet and dropping a ball from the same height at different points on the strings, the most powerful spot will return the ball to the highest height. Using the principles of impact and momentum, the coefficient of restitution between the ball and strings can be computed. The second experiment is conducted by lightly securing the ends of the racquet, placing an accelerometer on the racquet, then hitting the racquet with an impact hammer. Comparing the frequency response functions when the racquet is hit in different places, one can determine nodes and mode shapes, thus concluding the spot with the least vibration. Students will calculate center of percussion using dynamics principles. Variations of these experiments can also be conducted to determine the sweet spot of a baseball or softball bat.

Engineering Principles: Students are introduced to the concepts of impact and momentum, modal analysis, mass moment of inertia. Students will apply these concepts to determine the location of the sweet spots and compare the rebounding distance of travel of the ball when hit at these locations.

Module 3: Drag coefficient of a golf ball, with and without dimples

In external flow of a fluid over a body, laminar boundary layers result in much higher drag coefficients than turbulent boundary layers. This phenomenon is the underlying scientific motivation behind a variety of sporting goods design features including dimpled golf balls, smooth ping-pong balls and fuzzy tennis balls. Under some conditions, such as those that occur when a golf ball is driven, the effect can be profound. Specifically, a perfectly smooth golf ball would travel roughly half the distance of its dimpled counterpart. Students will study the effect of dimples on the drag coefficient of a golf ball.

The objectives of this module are as follows: (1) Perform background research to determine the velocity of a golf ball upon impact and estimate the Reynolds number. (2) Develop an experiment using the 0-100 MPH wind tunnel to measure the drag coefficient vs. Reynolds number of a golf ball. (3) Repeat the experiment on a smooth, golf ball sized sphere. (4) Make a plot of drag coefficient vs. Re number for both experiments and interpret the results in terms their impact on the game of golf. (5) For a given impact velocity, numerically calculate the distance that a golf ball will travel in a vacuum, in air with dimples and in air without dimples.

Measurements and Calculations: Students will perform experiments using a 0-100 MPH, 12 "x12" cross section wind tunnel. The students will measure drag force on the golf ball by mounting the ball on a flexible beam, fitted with a linear displacement transducer. Students will also measure the pressure along the surface of a golf ball using a manometer tube system. Students will calculate Reynolds number and drag coefficient by varying velocity in the wind tunnel.

Engineering Principles: Students are introduced to the concepts of boundary layers, lift, drag and the importance of non-dimensional numbers and dimensional analysis. Students will apply these concepts to perform a numerical analysis on the flight of a golf ball and compare these results to those that would have been obtained using the typical assumptions employed in an introductory Physics course. 
Module 4: Why does a curve ball curve? Does a good fastball rise?

In baseball, a curve ball is thrown by imparting a high angular velocity on the ball in addition to its translational velocity. In the early half of the 20th century, there was still debate in the scientific community over whether a curve ball actually curved or whether it was an optical illusion. Anybody who ever tried to hit a curveball found this debate to be curious since it was obvious that a curve ball did indeed curve. It is now well known that a curveball curves because of an effect called the Magnus effect. This effect is a result of the no-slip condition at the surface of the spinning baseball, which results in a velocity differential between the two sides of the baseball. Because of the Bernoulli effect, the velocity difference results in a pressure difference, which causes the curveball to curve. And, indeed it does.

A good fastball is often said to actually rise. Is this possible? Typically, a fastball is thrown with backspin on the ball. Theoretically, the same Magnus effect described above can result in enough upward lift on a fastball to cause it to rise. The question is, can a human being impart enough backspin on a fastball to make it rise? So, students will examine the effect of angular rotation on the lift of a sphere.

The objectives of this module are to (1) Perform background research to determine the translational and angular velocities of a typical curveball and baseball. (2) Develop an experiment using the 0-100 MPH wind tunnel to measure the transverse force on a baseball as function of Re and angular velocity. Interpret the results in terms their impact on a curveball and fastball. (3) Using typical values for the angular and translational velocity of a fastball, determine whether a good fastball can actually rise.

Measurements and Calculations: Students will perform experiments using a 0-100 MPH, 12 "x12" cross section wind tunnel. The students will measure drag force on a golf sphere ball by mounting the spinning ball on a flexible beam, fitted with strain gages to measure the deflection in two axes. Students will calculate the drag force and lateral force as a function of Reynolds number and angular velocity

Engineering Principles: Students are introduced to the concepts of the Bernoulli equation, the no slip condition, lift, drag and the importance of non-dimensional numbers and dimensional analysis. Students will apply these concepts to perform a calculation to prove whether a fastball actually rises.

Module 5: Frisbee ${ }^{\mathrm{TM}}$ Aerodynamics with Application to Ultimate Frisbee

The aerodynamics and dynamics of Frisbee's has only recently received scientific analysis even though it's been used by millions as a toy and more recently in the sports of ultimate Frisbee and Frisbee golf.

The objectives are: 1) to determine experimentally the nondimensional lift, drag, and pitching moment coefficients, as well as upper and lower pressure distributions, for different disc shapes and sizes under rotating and non-rotating conditions. 2) determine the dependence of the force and moment coefficients will be determined as a function of the angle of attack for different flow and rotation speeds. The importance of the Frisbee's rotation on the lift, drag and moment coefficients will be determined. 3) compare experimental results against theoretical predictions made using an incompressible CFD (computational fluid dynamics) analysis and algorithm.

Measurements and Calculations: Frisbee's will be mounted to the shaft of an electric motor instrumented with load cells and positioned at different angles ranging from $-20 \mathrm{o}$ to $20 \mathrm{o}$ 
relative to the wind speed; wind speeds that range from 3 to $30 \mathrm{~m} / \mathrm{s}$ will be explored. Published work will guide the development of the experimental hardware and their experimental data on force and moment coefficients will be used for quantitative comparison.[5,6,7] A pressure sensitive paint will also be applied to the upper and lower Frisbee surfaces to quantify the surface pressure distributions using a CCD camera (calibrated against a standard load versus color). The pressure will then be integrated over the surface and the results compared with the magnitude of the lift and drag forces. Additional dynamic experiments will be conducted on Frisbees that are instrumented with a 3-D Analog Devices MEM's accelerometer and wireless RF data transmitter (and accompanying ground-based receiver and data acquisition system) to quantify the time dependent loads and moments on a Frisbee in-flight.

Engineering Principles: Students will apply the conservation of mass, momentum and energy equations appropriate for incompressible fluids to approximate the pressure distributions on the upper and lower Frisbee surfaces assuming the Frisbee is effectively a thin airfoil. Using a finite-element CFD algorithm, the radial and azimuthal velocity fields and pressure distributions will be calculated, the pressure distribution will be integrated over the surface and the lift and drag coefficients will determined numerically and compared against the measured experimental values. The importance of Frisbee shape, lip, flow Re, and Frisbee rotation rate on the force and moment coefficients will be highlighted.

\section{Module 6 - Biomechanics of Human Movement}

Throughout the years, sports performances have continued to improve not only due to advances in sports equipment, but in training the human body to execute a performance more effectively and efficiently. Advances in motion capture technology, electromyography (EMG) and computer processing speed as have helped athletes improve their performances.

The objectives of this module are as follows: (1) Perform background research on action potentials and muscle physiology. (2) Develop experiments to record and measure human motion, ground reaction forces and muscle activation through EMG. (3) Analyze the body as a dynamic system, tracking positions and determining joint angles. (4) Apply Newton's Laws and Euler equations to determine joint reaction forces and moments. (5) Determine muscle activity through use of EMG.

Measurements and Calculations: By placing reflective markers on body landmarks and recording motion with the use of high speed video cameras, position data is acquired. Joint forces and moments will be calculated for simple 2-dimensional motions in lower level courses and 3-D in senior and graduate level electives.

Engineering Principles: Students are introduced to the concepts of Newton's Laws and Euler equations. Students will apply these concepts to determine joint reaction forces and moments as well as qualitatively look at sports improvements and performances during motions such as running, swinging a tennis racquet or throwing a baseball. This module can also be combined with the metabolism module to study the efficiency of the body when comparing to similar movements, such as running with and without ankle weights.

Module 7: The Dangers of Overhydration

In the 2002 Boston Marathon, a female runner died from hyponatremia, which is the result of severe overhydration. When she became exhausted and ill during the race, emergency personnel assumed that she was dehydrated and treated her accordingly. She died immediately upon the administration of hypotonic fluids (water with dilute electrolytes). The subject of 
overhydration among marathon runners and other endurance athletes has received increasing attention in recent years. In a study published in the New England Journal of Medicine[8], researchers present stunning evidence of the magnitude of this problem. Analysis of blood samples collected from runners in the 2002 Boston Marathon suggests that 1,900 runners had dangerously low sodium levels and 90 runners had critically low sodium levels at the end of the race, putting them in risk of confusion, seizures and death.

Overhydration is a result of excessive fluid intake such as that which can occur during a marathon when a runner follows the mantra "drink before you feel thirsty". When the extracellular fluid is diluted, fluid becomes hypotonic with respect to the interior of the cell. Cell membranes are semipermeable and allow water to be transported freely across them in order to maintain osmotic equilibrium. Water flows into the cell by osmosis, leading to rapid swelling of the cell, which often results in bursting (lysis). Severe hyponatremia can be treated with the IV administration of hypertonic ( $3 \%$ saline) solution; ingestion of water can be fatal. In this module, students will explore the role of osmosis on hydration status in biological systems.

The objectives of this module are to (1) perform background research on osmosis in biological systems (2) explore quantitatively the effect of concentrations of different species (salts, sugars, and proteins) on osmotic pressure of a fluid (3) to determine the effect of solute concentration on the osmotic water flux across a membrane.

Measurements and Calculations: A simple experiment will be conducted using thin slices of carrots as a biological system. The carrots will be weighed before and after soaking in a hypertonic or hypotonic solution (e.g., water, saline, or sports beverage), and the rate of water transport into or out of the cell will be determined.

Engineering Principles: This module focuses on engineering topics associated with the process of osmosis: osmotic pressure, osmotic flux, and membrane permeability. Students will explore the driving force for osmosis, which is the difference in osmotic pressure $(\Delta \pi)$ across the membrane as a function of species concentration. If the solution on each side is dilute, as is often the case with biological systems, then the osmotic pressure difference is equal to $\mathrm{RT} \Delta(\Sigma \mathrm{Cj})$, where $\mathrm{R}$ is the universal gas constant, $\mathrm{T}$ is temperature, and $\mathrm{Cj}$ is the molar concentration of the jth species. They will determine the rate of volumetric flow of solvent across the membrane, which is equal to $\mathrm{kA}(\Delta \pi-\Delta \mathrm{P})$ where $\mathrm{k}$ is the osmotic permeability, $\mathrm{A}$ is the membrane area, and $\Delta \mathrm{P}$ is the hydrostatic pressure difference. From their data for carrots, they will be able to determine the osmotic permeability of the carrot cells.

\section{Module 8: Metabolism and Work}

The air we inspire (inhale) is approximately $21 \% \mathrm{O} 2$ and $79 \% \mathrm{~N} 2$ on a dry basis. After rapid gas exchange in the lungs, the expired (exhaled) gas contains approximately $75 \% \mathrm{~N} 2,16 \%$ $\mathrm{O} 2,4 \% \mathrm{CO} 2$ that saturated with water and at body temperature. $[9,10]$ Oxygen consumed during respiration is transported by blood to cells for energy production through the oxidation of carbohydrates and fats from food. The reaction stoichiometry and thermodynamics are well known, and the rate of energy production may be calculated from the rates of $\mathrm{O} 2$ and $\mathrm{CO} 2$ exchange.[26] This energy is used to maintain the function of the body (basal metabolism, typically about $60-70 \%$ of total energy expenditure) and to do external work (exercise, typically about $30-40 \%$ of total).

The human body doing exercise can be analyzed as a machine doing mechanical work. To do mechanical work such as bicycling or running, the body expends energy. The mechanical efficiency, $\eta$, of this human "machine" is expressed by the following equation: 


$$
\eta=\frac{\text { mechanical work done }}{\text { energy consumed }} \cdot 100
$$

Measurements and calculations: A portable metabolic testing system will be used to obtain measurements of inspired and expired $\mathrm{O} 2$ and $\mathrm{CO} 2$ concentrations and average volumetric ventilation flow rate. Data will be collected during various exercise levels and at rest. The rates of oxygen consumption and carbon dioxide production will be calculated by mass balance. This information will allow the determination of the rate of energy expenditure by energy balance. Next, the rate of external work done during the particular exercise activity will be determined. Finally, the efficiency of the human machine performing a particular activity will be determined This analysis can be applied to any exercise or activity; provided it is possible to quantify the external work rate and that the activity is performed aerobically: This methodology will be used to determine the energy expenditure and human efficiency during aerobic weightlifting. In another module, we will investigate the effect of ankle weights on walking/running efficiency.

Engineering Principles: Students learn principles of stoichiometry, heat of reaction, mass and energy balances, work and efficiency.

\section{Engineering Principles in Sports Course at Rowan and Drexel Universities}

A new elective course will be developed as part of this project. Topics include aerodynamics; mechanics of sporting materials; mechanics of human movement; sports performance; dehydration and overhydration; ethical issues related to injuries, sport performance and product development, exploration of existing and new products and techniques, as well as encouraging innovation and entrepreneurship. Some of the modules described previously will be used for teaching students basic engineering principles. Students will be required to develop new experiments or make product modifications as part of a course project. This course will allow the students to develop the knowledge and skills necessary to become sports equipment designers and technologists and work with others in analyzing human performance.

\section{Integration of Sports Engineering in the Curricula}

\section{Freshman Engineering Clinic at Rowan}

Rowan's College of Engineering has developed an innovative curriculum built around the Engineering Clinics, an eight-semester sequence of courses in which every engineering student participates, starting with the first semester of the freshmen year. A key feature of the Clinics is the emphasis on multidisciplinary interactions - students from all four disciplines in the College of Engineering work collaboratively in teams, in the same way that industry operates.

The modules will be integrated into the Freshman Engineering Clinic course in Fall 2007. In the freshman year, the course objectives are to introduce students to the practice and profession of engineering, including team work, critical thinking, problem solving, technical communications, safety and professionalism. Embedded within these broader objectives is the goal to provide new engineering students with a survey of the different disciplines and to demonstrate the need for multidisciplinary collaboration. Based on our experience, the best way 
to achieve this is to employ hands-on exercises, which provide students with a context in which to learn. Thus, some of the modules described earlier are well suited for this purpose.

\section{Core course at Drexel, Gloucester County and Rowan}

The faculty at the participating institutions are developing the modules to share with one another and will use them in other relevant courses throughout the curricula besides those discussed above. Table 1 lists the modules and the courses in which they may be used. The reader may find this useful if he/she wishes to implement these modules in courses at his/her institution.

\begin{tabular}{|l|l|}
\hline Module & Course (Department) \\
\hline Mechanics of sporting materials & Solid Mechanics (CE, ME) \\
& Materials Science (ChE, CE, ME) \\
& Biomaterials (ME) \\
Biomechanics (ME)
\end{tabular}

Table 1: Modules and the relevant courses. Departments are Chemical Engineering (ChE), Civil Engineering (CE), Electrical and Computer Engineering (ECE), Mechanical Engineering (ME), and Health and Exercise Science (HES)

Summary

A set of hands-on modules are being developed that will be used in various courses throughout the curricula at three mid-Atlantic academic institutions. These modules will allow students to be engaged in their learning and discovery processes as they explore science and engineering principles with applications to sports.

Proceedings of the 2007 American Society for Engineering Education Annual Conference and Exposition Copyright (C) 2007, American Society for Engineering Education 
The topics introduced in these modules have been described and will be integrated throughout the engineering curriculum beginning in 2007. Plans for vertical integration include engineering and health sciences core courses, laboratory courses, and advanced senior and graduate level elective courses. In addition, a senior elective in "Engineering Principles in Sports" is being developed by faculty in Mechanical Engineering and Health and Exercise Science. Future work includes some assessments of student learning and use in outreach programs for K-12 students and teachers.

\section{Acknowledgments}

Funding for this project was obtained from the National Science Foundation, Division of Undergraduate Education (NSF DUE \#0536307). The authors would like to thank students Kyle Fitzpatrick, Kevin McGarvey, Robert Milane and Casey Oware for assistance in development and testing.

\section{References}

$1 \mathrm{http}: / / \mathrm{www} . a m e r i c a n s p o r t s d a t a . c o m /$ sports_injury1.asp (accessed May 2005)

2 http://www.prism-magazine.org/dec02/ballgame.cfm (accessed May 2005)

3 Rowan School of Engineering - A Blueprint for Progress, Rowan College, 1995.

4 Brody, H. Tennis Science for Tennis Players, University of Pennsylvania Press, 1987.

5 Stilley, G. (1972) "Aerodynamic analysis of the self sustained flair", RDTR no. 199, Naval Ammunition Depot, Crane, Indiana

6 Hummel, S. (1997) "Frisbee Flight Simulation and Throw Biomechanics," Master's Thesis, University of Missouri, Rolla

7 Mitchell, T.L. (1999) "The aerodynamic response of airborne discs", MS Thesis, University of Nevada, Las Vegas

8 Almond, C. The New England Journal of Medicine, Vol 352: pp 1550-1556, April 14, 2005

9 McArdle, W.D., F.I. Katch, and V.L. Katch, Exercise Physiology: Energy, Nutrition, and Human Performance, $4^{\text {th }}$ edition, Lea and Febiger, Philadelphia, PA 1996

10 Adams, Gene, Exercise Physiology Laboratory Manual, W.C.B. McGraw Hill, NY, 1998 